\title{
Rivaroxaban Failure in a SARS-CoV-2 (COVID-19) Positive Patient
}

\author{
Shwetha Gopal ${ }^{1 *}$, Krishna Kurpad ${ }^{1}$ and Geethika Earthineni2 \\ ${ }^{1}$ Rutgers Helath, Saint BARNABAS Medical Center, New Jersey, USA \\ ${ }^{2}$ Arkansas College of Osteopathic Medicine Center, New Jersey, USA \\ *Corresponding author: Shwetha Gopal, Rutgers Helath, Saint BARNABAS Medical Center, New Jersey, USA; Email: shwethag18@gmail.com
}

Received: August 16, 2021; Accepted: August 23, 2021; Published: August 30, 2021

\section{Introduction}

Atrial fibrillation is the most common sustained arrhythmia in clinical practice. Patients with atrial fibrillation are at increased risk of hospitalization, death, heart failure, and thromboembolic event [1]. Development and embolization of thrombus can occur with atrial fibrillation. Ischemic stroke is most common location of embolization of thrombus, although it can embolize to other locations [2]. Chronic oral anticoagulation is recommended in most of the valvular and non-valvular atrial fibrillation and should be started after careful consideration for embolic risk, bleeding risk and overall risks verses benefit should be assessed. Novel anticoagulants (NOAC) such as rivaroxaban, apixaban, dabigatran is recommended and are being increasingly used over warfarin for patients with non-valvular atrial fibrillation [3].

\section{Case Presentation}

This patient is an 85-year old female with an extensive past medical history of atrial fibrillation on rivaroxaban, breast cancer status post-mastectomy, dementia and hypertension presented to the ED from an assisted living facility with slurring of speech that started about 4 hours prior to presentation associated with confusion. Upon arrival to the emergency department, she was saturating $97 \%$ on room air and later required $2 \mathrm{~L}$ nasal cannula to maintain oxygenation above $92 \%$, heart rate of 70 beats per minute with an irregular rhythm, blood pressure of 147/63 mmHg was noted. On physical examination, she was alert, oriented to person and place but not time. She was not in any acute respiratory distress. Dry oral mucosa was noted. Lungs were clear to auscultation and S1 and S2 were heard. National Institute of health and stroke scale (NIHSS) score was 4 with not answering any questions scoring 2 , dysarthria as 1 , and right finger to nose dysmetria of 1. Cranial nerves and reflexes were grossly intact. Code stroke was activated, and the patient was immediately taken for a non-contrast Computer tomography (CT) scan of the head which revealed chronic microvascular and atrophic changes. Computer Tomography Angiography (CTA) was unable to be performed due to the infiltration of IV during the scan. The patient was deemed not a candidate for tissue plasminogen activator because she was out of the therapeutic window and no neurosurgical intervention was indicated because the patient was already on anticoagulation with rivaroxaban. Her white blood cell count was $14 \mathrm{~K} / \mathrm{CMM}$ with no neutrophilia or bands, Prothrombin time was 16 and the International normalized ratio (INR) was 1.4. Blood chemistry was within normal limits. Urinalysis was negative for leukocyte esterase or nitrites but positive for (+) 5700 bacteria. SARS-COV-2 PCR assay was positive. The patient was subsequently admitted to the medical floor. She was treated with ceftriaxone for 5 days for UTI. Magnetic Resonance Angiogram (MRA) of the brain revealed acute infarction of the left corona radiata and right frontal white matter. She was shifted from rivaroxaban to apixaban therapeutic dose for secondary prevention of stroke. Further hospital course remained uneventful and the patient was discharged back to assisted living. Follow-up after 2 months via phone call revealed that patient had no further episodes of stroke and her speech abnormality via therapy was gradually improving.

\section{Discussion}

Coronavirus (SARS-CoV-2) is a global health emergency affecting 33 million population with more than 1 milion deaths. Despite rapidly growing literature on COVID-19 inducing clinical procoagulant effect causing arterial and venous thromboembolism [4], exact pathophysiology of derangements in hemostatic system is not well elucidated. There is no evidence of intrinsic procoagulant effect of SARS-CoV-2. Most reasonable hypothesis is the inflammatory cascade activating coagulation pathway, similar to that observed in sepsis [5]. The mechanism of rivaroxaban failure in COVID positive patients is not well understood. Mechanism of action of rivaroxaban is dose dependent and compliance of medication, especially taken with food is increases the bio-availability especially at higher doses [6]. This patient was compliant with the medication and it was confirmed with the assisted living facility staff. Rivaroxaban is a competitive inhibitor of factor Xa, it binds directly and reversibly to factor Xa. It is metabolized through hepatic cytochrome system via CYP 3A4/5 and CYP2J2. One of the proposed mechanisms of rivaroxaban failure is coadministration of cytochrome inducers and thus reducing the bioavailability of the medication, resulting in treatment failure [7]. Mechanism of rivaroxaban failure in SARS CoV-2 is not well understood. There has been studies which has proved the apixaban is more effective in prevention of stroke in patients with atrial fibrillation [8]. Lack of laboratory availability to quantitatively measure rivaroxaban levels makes it difficult to determine the efficacy in this patient. Further research is required to develop a 
standard laboratory marker as either drug level or coagulation profile to identify the therapeutic level of the drug. There are no definite guidelines for treatment of patients with novel anticoagulation failure, further research is required to assess if switching to a different NOACs or different class of anticoagulation such as warfarin would be beneficial.

\section{Conclusion}

Rivaroxaban failure in patients with atrial fibrillation and COVID-19 positive should be recognized. Apixaban can be used as an alternative for secondary prevention of stroke.

\section{Financial Support and Sponsorship}

Nil.

\section{Conflict of Interest}

None declared.

\section{References}

1. Benjamin EJ, Wolf PA, D’Agostino RB, Silbershatz H, Kannel WB, et al. (1998) Impact of atrial fibrillation on the risk of death: the Framingham Heart Study. Circulation. 98: 946-952. [crossref]
2. Wolf P A, Abbott R D, Kannel W B (1991) Atrial fibrillation as an independent risk factor for stroke: the Framingham Study. Stroke 22: 983-988. [crossref]

3. Heidbuchel H, Verhamme P, Alings M, Antz M, Diener H-C, et al. (2015) Updated European Heart Rhythm Association Practical Guide on the use of non-vitamin K antagonist anticoagulants in patients with non-valvular atrial fibrillation. Europace 17:1467-1507. [crossref]

4. Bikdeli B, Madhavan MV, Jimenez D, Chuich T, Dreyfus I, et al. (2020) COVID-19 and Thrombotic or Thromboembolic Disease: Implications for Prevention, Antithrombotic Therapy, and Follow-Up: JACC State-of-the-Art Review. Journal of the American College of Cardiology. 75: 2950-2973. [crossref]

5. Iba T, Levy JH (2018) Inflammation and thrombosis: roles of neutrophils, platelets and endothelial cells and their interactions in thrombus formation during sepsis. Journal of Thrombosis and Haemostasis. 16: 231-241. [crossref]

6. J S, D K, M B, W M (2013) The effect of food on the absorption and pharmacokinetics of rivaroxaban. Int J Clin Pharmacol Ther. 51: 549-561. [crossref]

7. Kaur J, Rizvi S, Tewari P, Tamer S, Nafsi T (2016) Rivaroxaban Treatment Failure From Possible Drug Interaction: A Case Report. CHEST 149.

8. Fralick M, Colacci M, Schneeweiss S, Huybrechts KF, Lin KJ, et al. (2020) Effectiveness and Safety of Apixaban Compared With Rivaroxaban for Patients With Atrial Fibrillation in Routine Practice. Annals of Internal Medicine. 172: 463473. [crossref]

\section{Citation:}

